\title{
Características clínicas y microbiológicas de pacientes con periodontitis agresiva generalizada.
}

\section{Clinical and microbiological characteristics of a patients with generalized aggressive periodontitis.}

\author{
Jorge Patiño-Giraldo ${ }^{1 *}$, Carlos Ardila-Medina ${ }^{2}$ \\ 1. Universidad Antonio Nariño, Universidad de \\ Antioquia, Colombia. \\ 2. Universidad de Antioquia, Colombia. \\ * Correspondencia autor. Jorge Eliecer Patiño \\ Giraldo | e mail: cirujanojunior@gmail.com |Av. \\ Bolívar \#49 Norte -30 Armenia (Quindío), Colombia. \\ | FAX 057-6-7494981| \\ Trabajo recibido el 27/06/2017. Aprobado para su \\ publicación el 10/09/2017

\begin{abstract}
RESUMEN
Objetivo: el objetivo de este estudio es describir las características clínicas y microbiológicas de una muestra de pacientes diagnosticados con periodontitis agresiva generalizada (PAgG).

Materiales y métodos: En este estudio de corte transversal, 20 sujetos menores de 30 años con PAgG atendidos en las clínicas odontológicas de la Universidad de Antioquia en Medellín Colombia, fueron invitados a participar entre diciembre del 2015 y marzo del 2017, las muestras microbiológicas fueron analizadas usando técnicas de cultivo y tomadas en los seis sitios más profundos de cada paciente $(\geq 5 \mathrm{~mm})$.

Resultados: Prevotella ssp y F. nucleatum fueron detectados en altos porcentajes, además de los microorganismos comúnmente estudiados, el $10 \%$ de los pacientes fueron positivos para bacilos entéricos gram-negativos.

Conclusiones: se observaron grandes proporciones de microorganismos que incluyeron Prevotella spss y $F$. nucleatum; el $10 \%$ de los pacientes fueron positivos para bacilos entéricos gram-negativos.
\end{abstract} \\ Porphyromonas gingivalis (P.g) fue positivo para la mitad de los sujetos estudiados;

\section{PALABRAS CLAVE}

Periodontitis agresiva generalizada; Porphyromonas gingivalis, Aggregatibacter actinomycetemcomitans, bacilos entéricos Gram-negativos.

Rev. Clin. Periodoncia Implantol. Rehabil. Oral Vol. 10(3); 153-155, 2017.

\section{ABSTRACT}

Aim: The aim of this study is to describe the clinical and microbiological characteristics of a sample of patients diagnosed with generalized aggressive periodontitis (PAgG). Materials and methods: In this cross-sectional study, 20 subjects under 30 years of age with PAgG treated at the dental clinics of the University of Antioquia in Medellín, Colombia were invited to participate between December 2015 and March 2017, the microbiological samples analyzed using culture techniques were taken at the six deepest sites of each patient $(\geq 5 \mathrm{~mm})$.

Results: Prevotella ssp and F. nucleatum were detected in high percentages, Porphyromonas gingivalis (P.g) was positive for half of the studied subjects; In addition to the microorganisms commonly studied, $10 \%$ of the patients were positive for gramnegative enteric bacilli.

Conclusions: large proportions of microorganisms were observed, including Prevotella spss and F. nucleatum; $10 \%$ of the patients were positive for gram-negative enteric bacilli.

\section{KEYWORDS}

Generalized aggressive periodontitis; Porphyromonas gingivalis, Aggregatibacter actinomycetemcomitans, Gram-negative enteric bacilli.

Rev. Clin. Periodoncia Implantol. Rehabil. Oral Vol. 10(3); 153-155, 2017.

\section{INTRODUCCIÓN}

La periodontitis se caracteriza por la inflamación y la pérdida de los tejidos de soporte dental ocasionada por la alteración en la relación entre los microorganismos subgingivales y la respuesta inmune del huésped. ${ }^{(1)}$ Abarca dos manifestaciones principales: periodontitis crónica y periodontitis agresiva (PAg). En la periodontitis crónica se observa una prevalencia mayor a nivel mundial (1-20\%) comparada con la PAg (0.1-5\%). ${ }^{(2)}$ Sin embargo, es importante estudiar la PAg porque a pesar de su baja ocurrencia posee un alto riesgo de progresión y una pérdida de inserción marcada que concluye con la pérdida temprana de dientes y con secuelas alveolares importantes. ${ }^{(3)}$

La PAg se define como una forma de enfermedad periodontal de rápido progreso, predominante en sujetos jóvenes y generalmente sanos sistémicamente, ${ }^{(4)}$ clínicamente se clasifica como localizada cuando afecta al menos dos dientes permanentes, primeros molares e incisivos con al menos un sitio con profundidad de sondaje (PS) y nivel de inserción clínica (NIC) 
mayor o igual a $5 \mathrm{~mm}$, y no más de dos dientes diferentes a estos. Se considera generalizada cuando afecta al menos tres dientes permanentes diferentes de primeros molares e incisivos con al menos un sitio con profundidad de sondaje (PS) y nivel de inserción clínica (NIC) mayor o igual a $5 \mathrm{~mm} .^{(5)}$ Otra característica clínica importante es la destrucción periodontal severa a pesar de las cantidades relativamente bajas de depósitos bacterianos.

La periodontitis agresiva generalizada (PAgG) se considera una infección poli-microbiana, ${ }^{(1,6)}$ incluso la composición subgingival de la microbiota puede variar ampliamente entre distintos individuos y entre distintos grupos étnicos y geográficos. ${ }^{(7,8)}$ Los microorganismos patogénicos no actúan cada uno por sí solo, existe una relación e interacción dentro de la biopelícula y esta interacción favorece la colonización y la expresión de las propiedades bioquímicas y fisiológicas de las bacterias, que inducen la lesión en los tejidos de sostén.

En este orden de ideas es importante identificar características clínicas y microbiológicas de pacientes en diferentes poblaciones; en este caso, la caracterización clínica de pacientes con PAgG, en Medellín Colombia, aporta dilucidando nueva información para el diseño de mejores programas preventivos y para la elección de terapias farmacológicas específicas que permitan mejorar la respuesta de los pacientes al tratamiento.

\section{MATERIALES Y MÉTODOS}

En este estudio de corte transversal, 20 sujetos menores de 30 años con PAgG que fueron atendidos en las clínicas odontológicas de la Universidad de Antioquia en Medellín Colombia, fueron invitados a participar entre diciembre del 2015 y marzo del 2017. El consentimiento informado fue firmado por cada participante. El diseño del estudio fue aprobado por el comité de ética de investigación en humanos de la Sede de Investigación de la Universidad de Antioquia de acuerdo con las normas de investigación en seres humanos según lo dispuesto en la resolución No 008430 de 1993 del Ministerio de Salud y en la Declaración de Helsinki revisión de Fortaleza 2013, además será conducido de acuerdo a las líneas guías de buenas prácticas clínicas (GCP-ICH).

La población blanco del estudio comprende a los sujetos que padezcan periodontitis agresiva. La población se obtuvo de los pacientes que acudieron a la clínica de la Facultad de Odontología de la Universidad de Antioquia y que fueron diagnosticados con PAgG según los criterios establecidos por la Academia Americana de Periodoncia. ${ }^{(5)}$ Solo se incluyeron en el estudio los pacientes que cumplieron con los criterios de elegibilidad y aceptaron participar en el estudio firmando el consentimiento informado.

Los criterios de inclusión fueron: pacientes con edad menor o igual a 30 años, con al menos 20 dientes presentes, descartando terceros molares y dientes con extracción indicada, mínimo seis dientes permanentes con al menos un sitio con profundidad de sondaje (PS) y nivel de inserción clínica (NIC) mayor o igual a $5 \mathrm{~mm}$, incluyendo primeros molares o incisivos; y un mínimo de seis dientes diferentes de primeros molares e incisivos, con al menos un sitio con PS y NIC mayor o igual a $5 \mathrm{~mm}$, reporte de agregación familiar de periodontitis durante la anamnesis

Los criterios de exclusión fueron: pacientes embarazadas, lactantes o fumadores, pacientes bajo tratamiento con antibióticos o antiinflamatorios en los seis meses previos al estudio, pacientes que recibieron tratamiento periodonta en los seis meses previos al estudio, pacientes con diabetes, enfermedad cardiovascular, alteraciones inmunológicas, o cualquier enfermedad que pueda alterar el curso del tratamiento periodontal.

Evaluación clínica: a cada paciente se le realizó historia clínica con periodontograma y radiografías. Durante la anamnesis se le preguntó a cada sujeto si tenía al menos otro familiar con historia de periodontitis, para corroborar agregación familiar de la enfermedad. La presencia de placa (PB), sangrado al sondaje (SAS), PS y NIC fueron medidos en seis sitios por diente (mesio bucal, bucal, disto bucal, disto lingual, lingual y mesiolingual) en todos los dientes excluyendo terceros molares. La PS y NIC fueron medidas al milímetro más cercano usando una sonda estándar calibrada (UNC, Hu-Friedy, Chicago, IL). Las medidas fueron realizadas por el mismo periodoncista, entrenado calibrado.

Muestreo microbiológico: Las muestras microbiológicas de los pacientes con periodontitis fueron tomadas en bolsas mayores o iguales a $5 \mathrm{~mm}$. Se tomaron las muestras de los sitios más profundos de cada sextante. Se removió la placa supragingival con curetas y se aisló el área con rollos de algodón para posteriormente introducir las puntas de papel estériles \#30 (Maillefer, Ballaigues, Switzerland) dentro de cada bolsa periodontal durante 20 segundos. Las puntas de papel se transfirieron a un tubo de ensayo con $1 \mathrm{ml}$ de medio de transporte VMGA III bajo condiciones anaeróbicas e inmediatamente se envió al laboratorio de microbiología. El aislamiento de los microorganismos se hizo por métodos previamente reportados ${ }^{(9)}$. Las muestras se analizaron por medio de técnicas de cultivos microbiológicos para la presencia de bacterias periodontopatogénicas. Todas las muestras fueron procesadas antes de una hora a temperatura ambiente e inmediatamente incubadas en $\mathrm{CO}_{2}$ y sistemas de cultivo anaeróbico. El agar sangre fue incubado a $35^{\circ} \mathrm{C}$ en una jarra anaeróbica por siete días. La Identificación presuntiva se hizo de acuerdo a métodos ya descritos ${ }^{(10)}$ y usando un sistema de identificación comercial (RapID ANA, Remel, Norcross, GA, USA) para $A$. actinomycetemcomitans, $P$. gingivalis y $T$. forsythia. Cada paciente proporcionó un pull de muestras de placa subgingival.

Muestreo bacilos entéricos: Las puntas de papel se transfirieron a un tubo de ensayo con $2 \mathrm{ml}$ de medio de transporte VMGA III los frascos de la muestra se mantuvieron a temperatura ambiente y fueron transferidos al laboratorio y procesados dentro de las 4 horas posteriores al muestreo. Después los viales se colocaron en una incubadora durante 30 minutos a $37^{\circ} \mathrm{C}$, la placa bacteriana se dispersó mecánicamente con un agitador de tubos de ensayo en el ajuste máximo durante 60 segundos. Se prepararon diluciones en serie de 10 veces en agua de Pepton, y se colocaron en agar MacConkey. Las placas se incubaron aeróbicamente a $37^{\circ} \mathrm{C}$ durante 24 horas. Cada aislamiento se caracterizó según la morfología de la colonia y la coloración Gram. Los Bacilos entéricos gramnegativos fueron clasificados mediante una prueba bioquímica normalizada. El conteo se definió como el número total de unidades formadoras de colonias obtenidas en medios no selectivos. ${ }^{(11,12)}$

Análisis estadístico: Los datos fueron ingresados en una base de datos de Excel (Microsoft Office 2007) y se verificaron para evitar errores de ingreso. La unidad para el análisis estadístico básico fue el sujeto. Todo el manejo de datos y pruebas estadísticas se realizó usando un paquete de software estadístico (SPSS, Statistical Package for the Social Sciences, version 20, Chicago, IL). Los promedios, las desviaciones estándar, serán calculados para la descripción de los datos.

\section{RESULTADOS}

Treinta y ocho sujetos fueron evaluados para entrar en el estudio, de los cuales 18 fueron excluidos por no cumplir los criterios de selección, la tabla 1 muestra las características sociodemográficas y los parámetros clínicos de 20 pacientes; se observó un número mayor de mujeres (65\%). EI NIC y la PS fueron de $3.5 \mathrm{~mm}$ en promedio; la PB se encontró en $14 \%$ y el SAS en $32 \%$.

Tabla 1. Características demográficas y parámetros clínicos de pacientes con periodontitis agresiva generalizada.

\begin{tabular}{|c|c|}
\hline Parámetro & Sujetos PAgG $(n=20)$ \\
\hline Edad (años \pm SD) & $26 \pm 4,4$ \\
\hline femenino & $65 \%$ \\
\hline masculino & $35 \%$ \\
\hline NIC ( mm $\pm S D)$ & $3,5 \pm 0,6$ \\
\hline PS $(\mathrm{mm} \pm \mathrm{SD})$ & $3,5 \pm 0,5$ \\
\hline SAS ( mm $\pm S D)$ & $32 \%$ \\
\hline $\mathbf{P B}(\mathrm{mm} \pm \mathrm{SD})$ & $14 \%$ \\
\hline
\end{tabular}

$P B=$ placa bacteriana. $N I C=$ nivel de inserción clínica. $P S=$ profundidad al sondaje. $S A S=$ sangrado al sondaje. $S D=$ desviación estándar.

Los porcentajes de pacientes positivos para los microorganismos estudiados se muestran en la tabla 2. Fusobacterium nucleatum (F.n) fue aislado en mayoría de los pacientes seguido por Prevotella spp que también se encontró en un alto porcentaje. Porphyromonas gingivalis (P.g) fue positivo para la mitad de los sujetos estudiados. Además de los microorganismos comúnmente estudiados, el $10 \%$ de los pacientes fueron positivos para bacilos entéricos gram-negativos.

Tabla 2. Número de sujetos positivos para Aggregatibacter actinomycetemcomitans (A.a), Porphyromonas gingivalis (P.g), Prevotella spp, Fusobacterium nucleatum (F.n) y Bacilos entéricos gram negativos.

\begin{tabular}{ccc}
\hline Microrganismo & Frecuencia & Porcentaje \\
A.a & 4 & $20 \%$ \\
P.g & 10 & $50 \%$ \\
Prevotella ssp & 12 & $60 \%$ \\
F.n & 17 & $85 \%$ \\
Bacilos entéricos & 2 & $10 \%$
\end{tabular}

\section{DISCUSIÓN}

La periodontitis agresiva es una enfermedad que a pesar de su prevalencia baja $(1 \text { y el } 5 \%)^{(2)}$ produce pérdida de dientes y de inserción en edades tempranas. (3) Es importante que el tratamiento de elección disminuya el avance de la enfermedad y permita controlar el aumento en los parámetros clínicos limitando los daños permanentes; por esta razón la caracterización microbiológica y clínica 
de la PAgG es fundamental. Además durante la evolución de la enfermedad periodontal la flora microbiana se hace más compleja y los microorganismos de complejo rojo y el $A$. actinomycetemcomitans, pasan a dominar el ecosistema. ${ }^{(8)}$ En cuanto a los pacientes de este estudio diagnosticados con PAgG el mayor porcentaje fueron mujeres (65\%) lo que coincide con lo mostrado por Gajardo et $a l,{ }^{(13)} \sin$ embargo Susin et $\mathrm{al}^{(7)}$ no encontró diferencias entre la frecuencia de género. En cuanto a los parámetros clínicos de PAgG se encontró en promedio $3.5 \mathrm{~mm}$ tanto de PS como de NIC mientras que otros autores reportan promedios de $4.5 \mathrm{~mm}$ para periodontitis crónica generalizada (PCG). La PB es notablemente más baja en la PAgG que en la PCG con porcentajes de $14 \%$ y $78 \%$ respectivamente. ${ }^{(14)}$ De manera similar los porcentajes de SAS son inferiores en la PAgG comparados con los encontrados en PCG (14\% para PAgG y $81 \%$ para P. Crónica). ${ }^{(15)}$ Los resultados microbiológicos muestran que e F. nucleatum fue aislado en un $85 \%$ en los pacientes con PAgG lo que contrasta con el 33\% reportado por Gajardo et al en el 2005.(13) Por otro lado este estudio no encontró una asociación estadísticamente significativa entre la presencia de $A$ actinomycetemcomitans y los valores de NIC y PS, contrario a lo encontrado por Hamlet et al en el 2001 que afirma que el $A$. actinomycetemcomitans disminuye su presencia al aumentar la profundidad de las bolsas ${ }^{(16)}$. En la presente investigación la prevalencia de A. actinomycetemcomitans fue de $20 \%$, similar a hallazgo reportado por Takeuchi et al(17) pero diferente a la prevalencia mostrada por Gajardo y Hamlet del 33\%. ${ }^{(13,16)}$ Estos resultados se pueden explicar debido a que la composición de la microflora subgingival difiere notablemente entre pacientes y grupos étnicos y geográficos. ${ }^{(7,8)}$

La comprensión de la forma como se organiza y cambia la flora microbiana subgingival en la PAgG promueve la elección de terapias antibióticas adjuntas a la terapia mecánica; antimicrobianos como la azitromicina o la doxiciclina sin efectos importantes sobre la flora subgingival no se deben contemplar para el tratamiento en pacientes con este tipo de periodontitis, ${ }^{(18,19)}$ por el contrario terapias como amoxicilina y metronidazol, ${ }^{(20)} \mathrm{o}$ la más recientemente propuesta moxifloxacina, ${ }^{(21)}$ muestran una eficacia importante en el manejo coadyuvante farmacológico de la PAgG. Diferentes reportes muestran la presencia de bacilos entéricos gram-negativos en bolsas de pacientes diagnosticados con periodontitis, ${ }^{(22,23)}$ en nuestro estudio $10 \%$ de los pacientes presentaron resultado positivo para estos microorganismos, lo que confirma la participación de bacilos entéricos en la conformación del ecosistema de la periodontitis, sin embargo no se ha esclarecido el papel de ellos en la patogenia de la enfermedad. La coagregación y colaboración entre microorganismos juega un rol clave en el desarrollo del ecosistema patogénico de la periodontitis, ${ }^{(24)}$ en este estudio se observó una asociación estadísticamente significativa entre la presencia de $P$. gingivalis y $F$. nucleatum, confirmando la función en la biopelícula del $F$. nucleatum promoviendo la congregación de $P$. gingivalis, como lo presenta también la revisión de Diaz en el 2012. ${ }^{(25)}$

En conclusión los resultados muestran la participación en grandes proporciones de microorganismos como la Prevotella spss y F. nucleatum en la etiopatogenia de la PAgG, el $10 \%$ de los pacientes fueron positivos para bacilos entéricos gram-negativos.

\section{CONFLICTO DE INTERÉS}

Los autores declaran que no existe conflicto de interés concerniente a esta investigación.

\section{FUENTE DE FINANCIAMIENTO}

La Facultad de Odontología de la Universidad de Antioquia financia este estudio.

\section{RELEVANCIA CLÍNICA}

Justificación científica para el estudio: La flora microbiana de la periodontitis agresiva generalizada varía de acuerdo a las características étnicas y geográficas de la población, la descripción de la naturaleza microbiológica de un grupo de pacientes de Medellín Colombia provee información importante para la caracterización y comprensión de la enfermedad. Resultados principales: los pacientes mostraron resultado positivo para diferentes periodontopatógenos, F. nucleatum, y Prevotella ssp tuvieron las mayores proporciones. Se encontró una relación estadísticamente significativa entre la presencia de P.gingivalis y F.nucleatum.

\section{CONSECUENCIAS PRÁCTICAS}

La relación entre la presencia de diferentes periodontopatógenos y el aumento de la progresión de la enfermedad muestra la importancia de la elección correcta de terapias farmacológicas adjuntas al tratamiento mecánico de la periodontitis agresiva

\section{AGRADECIMIENTOS}

Al laboratorio de anaerobios de la clínica León XIII de la Universidad de Antioquia.

\section{Bibliografía}

1. Armitage GC, Cullinan MP. Comparison of the clinical features of chronic and aggressive periodontitis. Periodontol 2000. 2010;53(1):12-27.

2. Albandar JM, Tinoco EMB. Global epidemiology of periodontal diseases in children and young persons. Periodontol 2000. 2002;29(29):153-76.

3. Kulkarni C, Kinane DF. Host response in aggressive periodontitis. Periodontol 2000 2014;65(1):79-91.

4. Teughels W, Dhondt R, Dekeyser C, Quirynen M. Treatment of aggressive periodontitis. Periodontol 2000. 2014;65(1):107-33.

5. Armitage GC. Development of a Classification System for Periodontal Diseases and Conditions. Ann Periodontol. 1999 Dec;4(1):1-6.

6. Socransky SS, Haffajee AD, Cugini MA, Smith C, Kent RL Jr. Microbial complexes in subgingival plaque. J Clin Periodontol. 1998;25(2):134-44.

7. Susin C, Albandar JM. Aggressive periodontitis in an urban population in southern Brazil. J Periodontol. 2005;76(3):468-75.

8. Könönen E, Müller H-P. Microbiology of aggressive periodontitis. Periodontol 2000 2014:65(1):46-78.

9. D'Ercole S, Catamo G, Tripodi D, Piccolomini R. Comparison of culture methods and multiplex PCR for the detection of periodontopathogenic bacteria in biofilm associated with severe forms of periodontitis. New Microbiol. 2008;31(3):383-91.

10. Slots, J., Reynolds HS Long-wave UV light fluorescence for identification of blackpigmented Bacteroides spp. J Clin Microbiol. 1982;16:1148-51.

11. Slots J. Rapid identification of important periodontal microorganisms by cultivation Oral Microbiol Immunol. 1986;1(1):48-57.

12. Slots J, Rams TE SS. In vitro activity of chlorhexidine against enteric rods, pseudomonads and acinetobacter from human periodontitis. Oral Microbiol Immuno. $1991 ; 6: 62-4$

13. Gajardo M, Silva N, Gómez L, León R, Parra B, Contreras A. Prevalence of periodontopathic bacteria in aggressive periodontitis patients in a Chilean population. $J$ Periodontol. 2005; 289-94.

14. Feres M, Soares GMS, Mendes JAV, Silva MP, Faveri M, Teles R, et al. Metronidazole alone or with amoxicillin as adjuncts to non-surgical treatment of chronic periodontitis: 1-year double-blinded, placebo-controlled, randomized clinical trial. J Clin Periodontol. 2012;39(12):1149-58

15. Sampaio E, Rocha M, Figueiredo LC, Faveri M, Duarte PM, Gomes Lira EA, et al. Clinical and microbiological effects of azithromycin in the treatment of generalized chronic periodontitis: a randomized placebo-controlled clinical trial: Azithromycin in the treatment of ChP. J Clin Periodontol. 2011;38(9):838-46.
16. Hamlet SM, Cullinan MP, Westerman B, Lindeman M, Bird PS, Palmer J, et al. Distribution of Actinobacillus actinomycetemcomitans Porphyromonas gingivalis and Prevotella intermedia in an Australian population. J Clin Periodontol. 2001;1163-71. 17. Takeuchi $Y$, Umeda M, Ishizuka M, Huang Y, Ishikawa I. Prevalence of Periodontopathic bacteria in Aggressive periodontitis patients in a Japanese population in a Japanese population. J Periodontol 1999;70(10):1460- 69.

18. Haas AN, Silva-Boghossian CM, Colombo AP, Susin C, Albandar JM, Oppermann RV, et al. Adjunctive azithromycin in the treatment of aggressive periodontitis: Microbiological findings of a 12-month randomized clinical trial. J Dent. 2012;40(7):556-63

19. Xajigeorgiou C, Sakellari D, Slini T, Baka A, Konstantinidis A. Clinical and microbiological effects of different antimicrobials on generalized aggressive periodontitis. J Clin Periodontol. 2006;33(4):254-64.

20. Keestra JA, Grosjean I, Coucke W, Quirynen M, Teughels W. Non-surgical periodontal therapy with systemic antibiotics in patients with untreated chronic periodontitis: a systematic review and meta-analysis. J Periodontal Res. 2015;50(3):294-314.

21. Ardila CM, Martelo-Cadavid JF, Boderth-Acosta G, Ariza-Garcés AA, Guzmán IC. Adjunctive moxifloxacin in the treatment of generalized aggressive periodontitis patients: clinical and microbiological results of a randomized, triple-blind and placebo-controlled clinical trial. J Clin Periodontol. 2015:42(2):160-8.

22. Guzmán IC, Romero HG, Ardila-Medina MC. Administración sistémica adjunta de moxifloxacina versus ciprofloxacina más metronidazol en el tratamiento de periodontitis crónica con presencia de bacilos entéricos Gram negativos: I. Efectos clínicos y microbiológicos. Rev Fac Odontol Univ Antioq. 2012; 23: 207-24.

23. Ardila CM, Fernández N, Guzmán IC. Antimicrobial susceptibility of moxifloxacin against gram-negative enteric rods from colombian patients with chronic periodontitis. J Periodontol. 2010;81(2):292-9.

24. Kowalski JAN, Górska R. Clinical and microbiological evaluation of biofilmgingival interface classification in patients with generalized forms of periodontitis. Pol J Microbiol. 2014;63(2):175-81.

25. Diaz PI. Microbial diversity and interactions in subgingival biofilm communities Front Oral Biol 2012;15:17-40 